\title{
“LO HORRIBLE TAMBIÉN PUEDE SER MARAVILLOSO”. BIENESTAR EN UN CENTRO COMUNITARIO Y TERAPÉUTICO DE LA CIUDAD DE BUENOS AIRES
}

\author{
MARIA SOLEDAD DEL RIO
}

\begin{abstract}
RESUMEN
Este trabajo se propone rastrear las nociones de "bienestar" presentes en un Centro Comunitario y Terapéutico de la ciudad de Buenos Aires destinado, entre otras actividades, a ofrecer ceremonias de ayahuasca y de tabaco. Para ello me centraré en el análisis de la categoría nativa de sentir. A su vez, siguiendo los postulados de Birgit Meyer, intentaré mostrar que estos significados de la noción de "bienestar" encierran un tipo particular de estética (aisthesis). Es decir, de un tipo particular de experiencia sensorial total del mundo y del conocimiento del mismo. De esta manera, intentaré mostrar que la "estética del bienestar" presente en la categoría nativa del sentir debe entenderse como el resultado de una relación entre humanos y no-humanos (las plantas de tabaco y de ayahuasca).
\end{abstract}

\section{PALABRAS CLAVE \\ Ayahuasca; Sentir; Bienestar; Estética.}

\section{"O HORRÍUEL TAMBÉM PODE SER MARAVILHOSO". BEM-ESTAR NUM CENTRO COMUNITÁRIO E TERAPÉUTICO DA CIDADE DE BUENOS AIRES}

\begin{abstract}
RESUMO
Este trabalho propõe-se rastrear as noções de "bem-estar" presentes em um Centro Comunitário e Terapêutico da cidade de Buenos Aires destinado, entre outras atividades, a oferecer cerimônias de ayahuasca e de tabaco. Para isso, vou me concentrar na análise da categoria nativa de sentir. Por sua vez, seguindo os postulados de Birgit Meyer, tentarei mostrar que os significados da noção de "bem-estar" encerram um tipo particular de estética (aisthesis). Ou seja, um tipo particular de experiência sensorial total do mundo e do conhecimento do mesmo. Desta maneira, tentarei mostrar que a estética do "bem-estar" presente na categoria nativa do sentir deve ser entendida como 0 resultado de uma relação entre humanos e não-humanos (as plantas de tabaco e de ayahuasca).

\author{
PALAVRAS-CHAVE
}

Ayahuasca; Sentir; Bem-estar; Estética.

\section{"THE AWFUL CAN ALSO BE WONDERFUL". WELFARE IN A COMMUNITY AND THERAPEUTIC CENTER OF THE CITY OF BUENOS AIRES}

\begin{abstract}
This work intends to trace the notions of "well-being" present in a Community and Therapeutic Center of the city of Buenos Aires directed, among other activities, to offering ayahuasca and tobacco ceremonies. I focus on the analysis of the native category of sentir (feeling). In turn, following the postulates of Birgit Meyer, I will try to show that the meanings of the notion of "well-being" enclose a particular type of aesthetics (aisthesis). That is, a particular type
\end{abstract}


of total sensory experience of the world and of its knowledge. I argue that the "aesthetics of well-being" present in the native category of sentir (feeling) should be understood as the result of a relationship between humans and non-humans (tobacco and ayahuasca plants).

\author{
KEYWORDS \\ Ayahuasca; Feeling; Well-being; Aesthetics.
“L'HORRIBLE PEUT AUSSI ÊTRE MERVEILLEUX". BIEN-ÊTRE DANS UN CENTRE COMMUNAUTAIRE ET THÉRAPEUTIQUE DE LA VILLE DE BUENOS AIRES

\title{
RÉSUMÉ
}

Mon objectif c'est de traquer par les notions de "bien-être" présentes dans un centre communautaire et thérapeutique de la ville de Buenos Aires, destinées entre autres activités à offrir des cérémonies d'ayahuasca et de tabac. Pour cela, je me concentre sur l'analyse de la catégorie native du sentiment. En suivant les postulats de Birgit Meyer, je vais essayer de montrer que ces significations de la notion de "bien-être" contiennent un type particulier d'esthétique (aisthesis). C'est-à-dire d'un type particulier d'expérience sensorielle totale du monde et de la connaissance du monde. De cette façon, je vais essayer de montrer que "esthétique du bien-être" présent dans la catégorie native du sentiment doit être compris comme le résultat d'une relation entre humains et non-humains (les plantes de tabac et d'ayahuasca).

\section{MOTS-CLÉS}

Ayahuasca; Sentiment; Bien-être; Esthétique. 


\section{INTRODUCCIÓN}

En los últimos años se ha vuelto moneda corriente la articulación entre lo que la literatura especializada menciona como "espiritualidades contemporáneas" con nociones de "bienestar" o "salud". Estas articulaciones se han visto reflejadas en estudios que han abordado esta relación (entre lo "médico-psicológico" y lo "espiritual"1) bajo las categorías de "terapias alternativas" (TONIOL, 2015) o "bienestar espiritual" (VIOTTI, 2018). Muchas veces, estas articulaciones nacen de los propios grupos con quienes estudiamos. Es decir, suelen ser conexiones dadas por los propios actores y que tomamos de nuestros registros de campo. Sea que nos centremos en instituciones o en individuos y sus redes y prácticas cotidianas, esta articulación aparece en la literatura como un aspecto compartido por todos los grupos o movimientos que las ciencias sociales han englobado bajo las categorías de "nueva era" o "espiritualidad contemporánea". Nos referimos a prácticas tan variadas como el yoga, reiki, meditaciones, ceremonias de ayahuasca (muchas veces también englobadas dentro de las categorías de "chamanimo" o "neo-chamanismo"), reflexología, acupuntura, entre otras.

Siguiendo esta lógica, el presente artículo se propone rastrear las nociones de "bienestar" que entran en juego dentro de un Centro Comunitario y Terapéutico de la ciudad de Buenos Aires destinado, entre otras actividades, a ofrecer ceremonias de ayahuasca. Centro que, dado a su cruce entre "vegetalismo amazónico" y ciertas corrientes de la psicología, también podría caer dentro de las categorizaciones académicas de "religiosidades contemporáneas", "terapias alternativas" o "nueva era". Antes de continuar, considero que este objetivo merece algunas aclaraciones previas.

En primer lugar, evitaré referirme al Centro Comunitario y Terapéutico como una "religiosidad contemporánea", una "terapia alternativa" y sobre todo como una práctica de la "nueva era". Esto no implica evitar situar al Centro dentro del contexto más amplio en el que se inserta. Para el caso de Argentina, estaríamos hablando de procesos de liberalización y democratización de la vida diaria iniciados en la década de 1980, acompañados de procesos de mercantilización de la vida social y de transformación del sistema de mediaciones de la industria cultural, lo que permitió una mayor difusión de estas actividades a partir de la década de 1990 (SEMÁN; VIOTTI, 2018). Esta evitación, es más bien una puesta en suspenso, al menos por el momento. La utilización de estas categorías analíticas exige la necesidad de revisarlas

\footnotetext{
${ }^{1}$ Esta articulación no es exclusiva de "las nuevas religiosidades" o "las terapias alternativas", sino también aparece presente, por ejemplo, dentro del catolicismo. Para ello ver, por ejemplo (OLMOS ÁLVAREZ, 2018).
} 
previamente y explorar las tendencias epistemológicas que se encuentran detrás de ellas y que las sustentan. Esta tarea requeriría el armado de un nuevo artículo y por razones de espacio y privilegio de otras problemáticas, dejaré esta cuestión para un futuro cercano².

En segundo lugar, he escogido utilizar la noción de "bienestar" (y no otra, como podría ser, por ejemplo, la noción de "salud") ya que es aquella utilizada por la mayoría de "los/las nativos/as" para referirse a los efectos que las ceremonias de ayahuasca tienen sobre sus vidas cotidianas. En este sentido, el objetivo del artículo implica rastrear los contextos en los cuales la palabra "bienestar" es utilizada por "los/las nativos/as". Es decir, no me limitaré a una definición lingüística, de diccionario, sino trataré dar cuenta del contexto social (PITT-RIVERS, 1973) en el cual esta palabra es utilizada. Esto és, el significado de la noción de "bienestar" (una noción en primer lugar "nativa") sólo podrá entenderse en acción y como resultado de una situación de encuentro con la planta de ayahuasca y de tabaco. Por otro lado, también es importante señalar que no existe un único significado, sino que este varía de acuerdo a las distintas personas y sus estilos de vida, experiencias con la ayahuasca y sus formaciones profesionales o académicas. Por lo tanto, el/la lector/a deberá tener en cuenta que los sentidos y significados que le presentaré no reflejan una visión única y esencialista, sino más bien una multiplicidad de voces. Entonces, será importante entender la reconstrucción de mi trabajo de campo al modo de una "ficción etnográfica" (STRATHERN, 1988). Es decir, como una reconstrucción con fines analíticos.

A su vez, siguiendo a Birgit Meyer, intentaré mostrar que estos significados de la noción de "bienestar" encierran un tipo particular de estética. Esta noción no será abordada como un debate restringido al campo de las artes sobre aquello que se considera "bello". Más bien, será entendida, como lo señala Meyer, en su sentido aristotélico (aisthesis). Es decir, designando nuestra capacidad corporal basada en la fuerza de nuestra psique para percibir objetos del mundo a través de nuestros cinco modos sensoriales (MEYER, 2018). De esta forma, la noción de estética será utilizada para referirse a la experiencia sensorial total del mundo y del conocimiento de él, en este caso particular, mediado por la experiencia de participar en una ceremonia de ayahuasca. En este sentido, los postulados del embodiment y particularmente del concepto de "modos somáticos de atención" resultan centrales para entender estas experiencias. Estos conceptos de Thomas Csordas serán tomados en dos sentidos. En primer lugar, como paradigma y orientación metodológica donde el trabajo de campo etnográfico es

\footnotetext{
${ }^{2}$ Esta cuestión ha sido tratado por antropólogas y cientistas sociales con gran experiencia en el campo. Por ejemplo, podríamos mencionar los siguientes trabajos que, a su manera, expresan la necesidad de rastrar los trasfondos de las categorías de "religión" y "creencia": (BELL, 2004); (STEIL; TONIOL, 2013); (LUHRMANN, 2015); (de la TORRE; MARTíN, 2016); (GIUMBELLI; TONIOL, 2017).
} 
realizado prestando atención a y con el cuerpo (el de la propia investigadora en diálogo con los cuerpos de los/as otros/as). Con respecto a esto, resulta muy esquemático el siguiente fragmento de Jackson citado en el texto de Csordas (2010):

"Reconocer la corporalidad de nuestro ser-en-el-mundo es descubrir un terreno común donde yo y otro somos uno, por lo cual al usar el propio cuerpo del mismo modo que otros en el mismo entorno uno se encuentra a sí mismo impregnado por una comprensión que puede luego ser interpretada de acuerdo con la propia costumbre o inclinación, y que aún así permanece asentada en un campo de actividad práctica y por eso se mantiene en consonancia con la experiencia de aquellos entre los cuales uno ha vivido" (JACKSON, 1989 apud CSORDAS, 2010, p. 102).

En segundo lugar, el paradigma del embodiment y el concepto de "modos somáticos de atención" aparecerán como conceptos teóricos destinados a explicar cómo se construye esta "estética del bienestar" en el Centro Comunitario.

A lo largo del presente artículo, intentaré mostrar que esta "estética del bienestar" se encuentra reflejada en la categoría nativa del sentir. De esta manera, para el análisis de la misma dividiré el artículo en tres secciones. La primera sección estará dedicada a una aproximación inicial a la categoría del sentir. Allí mostraré que la noción del sentir debe ser entendida como la relación y el encuentro entre las teorías y bibliografías que influenciaron a los fundadores del CCT con sus experiencias con ayahuasca (a partir de aquí me referiré a él como CCT). En la segunda sección, intentaré dar cuenta cómo esas reflexiones en torno al sentir, presentadas en la primera sección, van configurando un determinado "mito de origen". Es decir, una explicación narrativa acerca de por qué las personas se acercan al CCT a beber ayahuasca y cómo llegan a relacionar a la planta con las categorías de "bienestar" y de sentir. En la última sección, veremos cómo la categoría del sentir se encuentra estrechamente vinculada a la práctica a partir del relato de mi participación en una ceremonia de rape de tabaco. Aquí veremos cómo el "bienestar" es alcanzado a partir de sentir "malestares" físicos y cómo ambas nociones se encuentran vinculadas y entrelazadas.

Por último, antes de comenzar, quisiera mencionar dos cuestiones fundamentales. En primer lugar, quisiera realizar una aclaración con respecto a la metodología utilizada para el análisis de esta "ficción etnográfica". En este sentido, las reflexiones que presentaré en este artículo se encuentran basadas en mi trabajo de campo en el Centro Comunitario y Terapéutico ${ }^{3}$ de la Ciudad de Buenos Aires. El mismo fue iniciado en 2015 mientras me encontraba realizando mi tesina de grado y es continuado en la actualidad para la investigación realizada para mi futura tesis de Maestría del Programa de Posgrado del IDAES y el IDES de la Universidad Nacional de

\footnotetext{
${ }^{3}$ Para un paneo general acerca de las principales actividades del Centro Comunitario y Terapéutico ver: del Rio, M. Soledad (2018).
} 
San Martín. A lo largo de estos años, mi aproximación etnográfica ha consistido en: mi participación (observación-participante) en dos de las actividades del CCT (las meditaciones chamánicas y las ceremonias de ayahuasca), charlas informales con distintos participantes antes de iniciar o finalizar una meditación o una ceremonia de ayahuasca, toda la información presentada en el sitio web y en el Facebook del CCT y entrevistas etnográficas tanto a los fundadores del CCT como a los participantes de sus actividades que han aceptado charlar conmigo. Todo ello fue reconstruido y registrado en mis diarios de campo. Las conversaciones y escenas etnográficas aquí presentadas, por lo tanto, representan reconstrucciones hechas a posteriori luego del "estar ahí". Todo lo que identifico como "voces nativas" serán presentadas en itálicas dentro del cuerpo del texto.

En segundo lugar, quisiera presentar brevemente los principales objetivos del CCT y a sus principales participantes: sus fundadores y los/las ayahuasqueros/as.

El Centro Comunitario y Terapéutico nace como una asociación civil hace a aproximadamente 13 años a partir de la iniciativa de un matrimonio de psicólogos; la cual, a su vez, surge a partir del primer encuentro de uno de ellos con las ceremonias de ayahuasca. El CCT se presenta como un espacio ecléctico que a partir de la combinación de distintas técnicas de la psicología con el curanderismo amazónico (ceremonias de ayahuasca y soplado de tabaco) se propone brindar herramientas para que las personas puedan alcanzar su bienestar emocional y espiritual o, como suelen decir, conectarse con su sentir. Para ello ofrecen distintas actividades que son presentadas por ellos mismos como servicios. Entre ellas se encuentran las meditaciones chamánicas (que consisten en meditaciones guiadas por cantos y soplado de tabaco), las ceremonias de ayahuasca y las ceremonias de rape (son similares a las meditaciones chamánicas con la diferencia de que aquí se aspira rape de tabaco y se extiende su duración de una hora a tres). Esta categoría de servicios remarca dos cuestiones. Por un lado, que las actividades se organizan con un propósito (alcanzar el bienestar emocional y espiritual) y que están a disposición de todas aquellas personas que quieran participar de ellas. Por otro lado que, como todo servicio, implica una retribución. Todas las actividades, con la excepción de las ceremonias de ayahuasca que cuentan con un valor fijo, son retribuidas con una colaboración a voluntad en dinero. El cual es utilizado para sustentar el espacio: comprar ayahuasca en Perú, para el mantenimiento de la infraestructura como podría ser pintura, comprar algún instrumento musical nuevo que pueda servir para la meditación, los gastos de luz y agua, los insumos del baño (jabón para las manos, papel higiénico), baldes, la comida que se ofrece al finalizar una ceremonia, etc. 


\section{LOS FUNDADORES}

S y J están casados hace varios años y tienen dos hijas. S, una mujer de unos 55 años aproximadamente, estudió psicología en la Universidad de Buenos Aires (UBA) y se recibió en el año 1989. Ejerce su profesión hace aproximadamente 30 años aunque sus actividades dentro del Centro (como guía en las ceremonias de ayahuasca o como sopladora de tabaco en las meditaciones) están por fuera de sus actividades como psicóloga. Si bien se recibió en la UBA, sus inclinaciones se encuentran lejos del psicoanálisis de Freud y Lacan. Por el contrario, al igual que J, ha preferido la psicoterapia humanística, es decir, una psicoterapia que considera al ser humano como un ser global en el que convergen sentimientos, pensamientos, conductas y acciones y que privilegia un tipo de abordaje tanto individual como grupal. Por su parte J, un hombre de unos 60 años aproximadamente, estudió la misma carrera que se esposa pero varios años más tarde, recibiéndose en el 2001 en la Universidad de Flores. Además, J es analista en sistemas. De hecho éste constituye su trabajo principal, es decir, la fuente de ingresos para su familia.

Sy J se han acercado a la ayahuasca en momentos diferentes. J, se acercó por primera vez hace más de 12 años. Un compañero suyo de la facultad se encontraba organizando y oficiando ceremonias de ayahuasca y lo invitó a J para que participara de una toma. Después de esta primera experiencia, comienza a nacer en él un deseo de ayudar a las personas en su búsqueda de bienestar emocional y espiritual a partir de las tomas de ayahuasca. Dicho deseo se concretizó en la fundación del CCT y en la organización de ceremonias de ayahuasca. De esta manera, Jorge comenzó a viajar a un Centro de lquitos, Perú, manejado por un curandero indígena shipibo. Estos viajes, realizados una vez al año, tenían como objetivo la adquisición de ayahuasca y también el inicio de un proceso de aprendizaje de técnicas de curación con Plantas Maestras, entre las cuales la ayahuasca era la principal protagonista. De esta forma, la participación de curanderos peruanos representa para los fundadores del Centro el comienzo de un intercambio de experiencias y botellas de ayahuasca entre personas de distintas culturas.

S, por su parte, se acercó por primera vez a la ayahuasca en una ceremonia que ofrecieron en el CCT, ceremonia que había sido oficiada por Doña Asencia, una curandera shipibo de Perú que ha visitado en varias ocasiones al CCT. Según me comentó S, esta ceremonia fue muy importante para ella ya que le permitió trabajar la confianza. Según sus palabras, se consideraba una persona muy estructurada y comenzar un Centro destinado al bienestar a partir de la ingesta de ayahuasca le generaba muchas dudas en ese entonces. Dudas que deben entenderse en relación al hecho de que en Argentina la ayahuasca se encuentra situada dentro 
de un "gris legal". Su participación en esta ceremonia la convenció de que, con ayahuasca, tanto ella como cualquier otra persona que quisiera participar, podían cumplir los objetivos que se proponían como Centro Comunitario y Terapéutico.

\section{LOS/AS AYAHUASQUEROS/AS}

Bajo esta categoría intento definir a las personas que participan de las ceremonias de ayahuasca del CCT. Si partimos de la observación participante, podemos describir a estas personas como un grupo bastante heterogéneo en lo que refiere a género, edad, profesiones y trabajo. No obstante, para darle peso cuantitativo a esta afirmación, para mi futura tesis de maestría he realizado hasta el momento unos 66 cuestionarios de 10 preguntas cada uno los cuales se repartieron (a aquellos interesados en responder) todos los viernes al finalizar la meditación durante el 2016. El cuestionario es anónimo y cuenta con preguntas centradas en cuestiones biográficas (referentes al género, edad y profesión de la persona) y en las actividades (referidas a el primer acercamiento al CCT, el tiempo que llevan participando de sus actividades y a su participación en otras actividades "similares" en otros lugares). En resumen, gracias a este pequeño cuestionario puedo realizar una primera caracterización de las personas que asisten al CCT. De esta manera, puedo decir que mujeres y hombres de edades que oscilan entre los 13 y los 62 años de profesiones y oficios variados se han acercado al CCT a través de recomendación de un familiar y/o amigo o porque encontraron el centro por Internet, de los cuales más de la mitad ya habían participado de otras actividades perfiladas, por autores como Carozzi (2000), pertenecientes al "circuito alternativo": actividades tales como yoga; meditaciones; ceremonias de ayahuasca, hongos, San Pedro y wachuma, temazcales; danzas; reiki; sanación femenina; círculos femeninos; registros akashicos y canalizaciones. Cabe aclarar que a las ceremonias de ayahuasca sólo participan mayores de 18 años.

Habiendo presentado al CCT, sus fundadores y a los/las ayahuasqueros/as, pasaremos a continuación, al desarrollo de la categoría nativa del sentir.

\section{SIENTO, LUEGO EXISTO. UNA BREVE APROXIMACIÓN A LA CATEGORÍA NATIVA DEL SENTIR}

La primera vez que me acerqué al CCT, en abril del 2015, le pedí a J que me comente cómo fue su primer acercamiento a la planta de ayahuasca y cómo se fue formando el CCT. De este relato, para los fines de este artículo, quisiera resaltar el siguiente fragmento:

La gente busca soluciones, equilibrio emocional y satisfacción con lo que sentimos. Por eso la gente se acerca. Pero también están aquellos que sólo se acercan por la experiencia psicodélica. Esta es sólo una pequeñísima parte de lo que es la ceremonia. La ayahuasca nos 
conecta con el sentir, con las emociones, nos ayuda a resolver problemas que con la cabeza no podemos resolver. ¿Viste la frase esa, "Pienso, luego existo"? Bueno, yo diría más bien primero sentimos y después pensamos. La ayahuasca nos permite activar nuestro ser espiritual liberando nuestro potencial. Se trata de una experiencia grupal pero también íntima.

Este relato no sólo representa mi primer acercamiento a la noción de "bienestar" (aunque en ese momento inicial de mi trabajo de campo no lo había advertido así) sino que también resume la aisthesis del CCT. Vayamos desarmando estas ideas por partes. Aquí el fundador del CCT utiliza una noción que aparece como central y que engloba tanto la idea de "bienestar" como la de "estética" (y también la de "espiritualidad"): el sentir. Los sentidos que a lo largo de mi trabajo de campo pude ir registrando se encuentran estrechamente vinculados a la práctica. Se trata de una categoría que no cuenta con una definición formal sino más bien de pequeñas reflexiones que se comparten al finalizar las actividades del Centro. Pese a las variaciones que podamos encontrar entre una y otra persona (variaciones ligadas más bien a las experiencias de vida y a sus propias relaciones con la planta de ayahuasca) existen ciertas ideas compartidas en torno al sentir. Son estas reflexiones y su puesta en conjunto las que nos permitirán crear un sentido único o más bien general acerca de lo que es el sentir (otra vez hay que tener en cuenta que esta reconstrucción es más bien ficcional - en el sentido que $M$. Strathern da a la noción de "ficción etnográfica" - y, a su vez, es la reconstrucción que yo como antropóloga realizo a partir de mi experiencia de trabajo de campo con las personas que participan del CCT). Es decir, para entender los alcances de esta categoría debemos entender las relaciones que las personas entablan con la planta de ayahuasca y con el tabaco mapacho tanto en las ceremonias como en las meditaciones. Teniendo en cuenta estas cuestiones, pasemos a desentrañar la noción "nativa" del sentir.

En otro artículo (del RIO, 2018) mencioné que para entender esta categoría primero tenemos que remontarnos a las influencias teóricas de los fundadores del CCT. Influencias que, vuelvo a mencionar, se encuentran estrechamente relacionadas con las experiencias particulares con la ayahuasca y con sus estudios en el área de psicología. Me refiero aquí a ciertas corrientes de esta disciplina que, en cierto sentido, se distancian del psicoanálisis de orientación FreudianoLacaniano. Una de ellas, es la psicología practicada por Carl Gustav Jung. De este autor toman la noción de que el ser humano puede orientarse en el mundo a través de cuatro funciones básicas: el sentimiento, el pensamiento, la intuición y la sensación (GONZÁLEZ, 2004). Todas estas funciones representan una unidad que es el ser humano. Veamos como entra en juego esta definición en el Centro. Para el CCT nuestra cultura (la cual es entendida como "moderna y occidental") pone el acento en el pensamiento racional dejando de lado el desarrollo de las otras tres funciones señaladas por Jung. Este énfasis de nuestra cultura en el pensamiento racional 
constituye un grave problema para la socialización según afirman los fundadores del CCT. Y es en base a él que definirán el principal objetivo de sus actividades:

Nuestra misión es ayudar al ser humano a reconectarse con su emocionalidad porque está muy polarizado en la mente, la mente racional, la mente de la palabra, y necesita hacer crecer la otra parte [el sentir]. La otra parte es lo que te permite decidir qué camino tomar para sentirte bien. Pero la realidad es que estamos hablando que hoy en día hay mucha crisis; mucha crisis porque las personas tratan de manejar sus emociones con la cabeza. Y son momentos para poder recuperar el sentir para poder elegir lo que uno quiere en base al sentir.

Aquí aparece otra cuestión clave. Para entender el significado del sentir hay que contraponerlo en oposición al pensamiento racional. Pero también hay que tener presente que esta contraposición y oposición implica una complementariedad entre ambos - el pensamiento racional y el sentir. Es decir, para el CCT el sentir no viene a reemplazar al pensamiento racional, más bien viene a formar parte de él, a servirle de guía, de brújula. Ambos, pensamiento y sentir son partes constitutivas del ser.

El pensamiento es una herramienta importantísima pero que por sí sola no basta. Tiene que estar al servicio del sentir. El sentir es aquello que no se puede explicar, porque cuando se explica deja de ser lo que es. Es simple, es sentir las sensaciones del cuerpo. Y el sentir tiene que guiar el pensamiento. El pensamiento está al servicio del sentir (palabras de la fundadora del CCT).

El pensamiento racional, entonces, pertenecería al plano de las representaciones y constituye una noción que para el CCT trae aparejada una división, en términos cartesiana, entre mente (pensamiento racional) y cuerpo (como aparato biológico). A partir de la experiencia con ayahuasca, esta división comienza a diluirse y a re-significarse. Aquello que nuestra cultura entiende como separado, la ayahuasca muestra como unidad. Para el CCT, así como para la mayoría de las personas que participan de las tomas de ayahuasca, el ser humano ya no es más una división entre mente y cuerpo. Es una unidad que engloba a ambos. El cuerpo ya no es un mero aparato biológico, sino que se convierte en medio y fuente de conocimiento. Y la categoría que utilizan para dar cuenta de esto es la del sentir. De esta forma, estas categorías de mente y cuerpo son englobadas en la de sentir que da cuenta tanto de las ideas y pensamientos como de las emociones y los sentimientos así como también de los procesos del cuerpo mismo (entendidos como los efectos fisiológicos ${ }^{4}$ de la ayahuasca sobre éste: mareo, malestar

${ }^{4}$ El biólogo Richard Evans Schultes (2000) destacaba que los efectos de la bebida varían según el método de preparación, el contexto en el cual se toma, la cantidad ingerida, el número y tipo de mezclas, el propósito con el cual se usa, así como el control ceremonial que ejerce el chamán. La ingestión de ayahuasca por lo general produce náusea, vértigo y vómito y lleva tanto a estados eufóricos como agresivos. Este autor afirma que la intoxicación con ayahuasca produce visiones luminosas después de un período de vértigo, nerviosismo, profusa transpiración y algunas veces náuseas. También menciona que un efecto secundario muy frecuente después de la intoxicación es la diarrea. Las triptaminas presentes en el brebaje intensifican muchos de estos efectos; aparecen, además, palpitaciones, convulsiones, midriasis y taquicardia. Por otro lado, estudios más recientes 
estomacal, vómitos, vibraciones en todas las partes del cuerpo, sensación de cosquilleo, cambios en la temperatura corporal).

Para el CCT no sería la razón aquello constitutiva del ser humano, sino justamente el sentir, una noción que les parece mucho más elemental y esencial que la noción cartesiana de razón a la que estamos acostumbrados (nosotros los "modernos occidentales"). El sentir remite a la experiencia, por eso siempre mencionan el hecho de que resulta difícil ponerla en palabras. Y esta experiencia es la experiencia del cuerpo (el cuerpo como sentimientos, como emociones, como biológico y como pensamientos y reflexiones). Por esta razón, afirman que el sentir nos recuerda que constituimos una totalidad con la naturaleza y que, a su vez, como seres humanos representamos una totalidad en sí mismos.

Teniendo presente lo expuesto en el párrafo anterior, pienso que las ideas que consideran a este tipo de prácticas como "una versión softcore de la psicodelia de los sesenta y de ambigua pertenencia contracultural, que detrás de una impronta espiritual transformadora esconde un individualismo consumista, producto de una sociedad tecnificada y capitalista" (BAER; MORRIS apud APUD, 2013), no sólo son injustificadas y contradichas por los datos empíricos, sino también etnocéntricas. No sólo oscurecen las redes de relaciones y prácticas que las personas tejen (de una manera muy enmarañada) en sus vidas cotidianas, sino también los aspectos positivos que encuentran en ellas. Quizá más que hablar de "individualidades", podríamos hablar de "singularidades". Porque esta noción de "singularidad" (DUARTE, 2013) refleja la interrelación entre cada persona en singular (con su historia de vida personal) con el grupo en su totalidad, con el cual sienten empatía y pertenencia ya que representan una "totalidad-unidad" que se refleja en esa frase que suelen repetir continuamente todos somos uno; somos parte de lo mismo. Con respecto a esta cuestión de la "singularidad" y la "unidad", por ejemplo, un visitador médico de unos 57 años aproximadamente me comentaba, en una charla grabada que tuvimos en mayo del 2016, que

Entonces, acá hay una conexión entre todos. Y eso lo sentís, que es un amor fraternal. (...) Lo interesante de esto es que vos observas que todos pueden ver algo que pertenece a una cosa muy profunda y después cada uno vive lo suyo. Para mí es como un salto cuántico, poder estar dentro de uno ver su mundo y a la vez poder observar otros mundos. (...) Es un mundo

desde la neurociencia (KUYPERS; RIBA; de la FUENTE REVENGA; BARKER; THEUNISSEN; RAMAEKERS; 2016) comentan, citando otros trabajos, que la Encuesta Nacional sobre Uso de Drogas y Salud en los EEUU reveló que el uso psicodélico clásico de por vida se asoció con una reducción significativa en la angustia psicológica y el pensamiento suicida (KUYPERS et al. 2016). También mencionan que se ha sugerido que los psicodélicos como la ayahuasca podrían ser, dentro de un entorno psicoterapéutico apropiado, útiles en el tratamiento de los trastornos por uso de sustancias, ya que pueden provocar cambios de estilo de vida positivos y duraderos (KUYPERS et al. 2016). Por último, estos autores citan que un estudio reciente mostró que la ayahuasca mejora las capacidades relacionadas con la atención plena, un objetivo clásico de ciertas intervenciones psicoterapéuticas (KUYPERS et al. 2016). 
compartido, y ese mundo compartido tiene que tener una esencia, algo superior, algo que está más allá de todos. Y esa es la reunión.

A modo de cierre, podemos decir que el sentir no se encuentra en una parte específica del cuerpo. No hay un dónde o un cómo sentir; sentir es más bien la experiencia del "cuerpo vivido". Un cuerpo que ya no es sólo biológico y que se encuentra separado del pensamiento. El cuerpo del sentir, por el contrario, es experiencia y esto implica que se siente, se percibe y se conoce con el cuerpo y desde el cuerpo. Como menciona Thomas Csordas retomando a MerleauPonty: "la percepción comienza en el cuerpo y, a través del pensamiento reflexivo, termina en los objetos. En el nivel de la percepción, no hay una distinción sujeto-objeto - simplemente somos en el mundo" (CSORDAS, 2010, p. 85).

Ahora bien, aquello que se siente, se percibe y se conoce, por un lado, va a depender de cada persona singular, de su historia de vida, de su personalidad y su experiencia con ayahuasca o con tabaco (o con ambas). No obstante, el sentir se extiende y expande hacia los otros cuerpos. Hay aquí una "educación de la atención" (INGOLD, 2015) en y hacia el sentir. Se aprende y se educa al cuerpo a prestar atención a estas sensaciones, emociones y pensamientos de manera colectiva. O en términos de Thomas Csordas, el sentir educa hacia los "modos somáticos de la atención". Estos son "modos culturalmente elaborados de prestar atención a, y con, el propio cuerpo, en entornos que incluyen la presencia corporizada de otros" (CSORDAS, 2010, p. 87). Según este autor, prestar atención a una sensación corporal no es prestar atención al cuerpo como un objeto aislado, sino que es prestar atención a la situación del cuerpo en el mundo (CSORDAS, 2010). A su vez, afirma que uno presta atención con su propio cuerpo; y esto es más bien un compromiso corporal que una función cognitiva. De esta forma, prestar atención a nuestro propio cuerpo puede decirnos algo sobre el mundo y sobre los otros que nos rodean. Estos modos no son ni arbitrarios ni biológicamente determinados, sino que están culturalmente construidos (CSORDAS, 2010).

Veamos cómo son construidos estos "modos somáticos de atención" por el propio CCT. Por un lado, este aprendizaje es con la planta de ayahuasca; por el otro, es con todo el grupo (los fundadores del CCT y las personas que están participando de la ceremonia). El CCT pone énfasis no sólo en que las personas presten atención a su respiración durante una meditación o una ceremonia, a lo que les pasa en el cuerpo mientras están en ellas, a su sentir en tanto singularidad (¿se duerme alguna parte del cuerpo? ¿cuál es la posición dónde estoy más cómodo/a?, ¿qué emociones producen las visiones de ayahuasca? ¿qué muestran estas visiones? ¿qué pasa con el cuerpo a nivel fisiológico: estoy mareado/a, tengo náuseas, estoy vomitando? ¿qué pensamientos vienen durante la meditación o la ceremonia? ¿qué sensaciones me produce 
el otro: su respiración, su movimiento, su llanto, su risa, su vómito, sus náuseas, etc.). Sino que también habilitan espacios, generalmente al final de una ceremonia o de una meditación, donde estas cuestiones son compartidas con los demás participantes. Así, en el diálogo y en el discurso de sus fundadores, se va construyendo esta categoría colectiva del sentir y se va educando el cuerpo (y el pensamiento) en una dirección. Es decir set (los determinantes internos de la persona en tanto singularidad) y setting (el entorno o contexto socio-cultural dado por la estructura ritual de las ceremonias y por los discursos de sus propios fundadores) están moldeando y posibilitando la construcción y la educación del sentiry, por lo tanto, las reflexiones acerca de las experiencias con ayahuasca y tabaco.

Pasemos a continuación al armado del setting, o "mito de origen" que sustenta y moldea las experiencias corporalizadas del sentir.

\section{EL CAMINO DEL SER. LA CONSTRUCCIÓN DEL MITO DEL HÉROE.}

Nosotros representamos arquetipos. Y los arquetipos son como los causes de un río. No se ven pero modelan la vida de la humanidad. Si vos ves distintas culturas, bueno, lo que ves en común en todas las culturas, eso son los arquetipos. Fenómenos inconscientes. Y fueron representados, por ejemplo, como el mito del héroe. Como la Odisea. Si vos lees, es todo el camino del ser, la pérdida de identidad y todo el recorrido para recuperar el alma. A través de un camino, sí, espiritual. Y esos mitos los representamos todo el tiempo. Primero viene la caída. Después uno va recorriendo por distintas experiencias que son frustrantes. De repente uno es sacudido por cuestiones y de repente tiene que liberarse. Y de repente llega el destino. Vuelve transformado. Es lo que pasa. Ese mito del héroe es un buen mito porque es la representación de nuestra vida (...). O sea, el mito no es la parte fantástica de la historia. El mito es el proceso viviente de tu forma de moverte en la vida.

Estas fueron las palabras de J, el fundador del CCT en una charla grabada por Julio en 2015. Este fragmento muestra cómo la "estética" ayahuasquera también tiene su propio mito de origen. La experiencia con ayahuasca puede ser entendida como ese camino del ser o mito del héroe que señala el fundador en su relato. En este sentido, podemos decir que una persona se acerca por primera vez a la ayahuasca porque sufrió una pérdida de identidad (como vimos en la sección anterior esta pérdida de identidad estaría ligada a la idea de la preeminencia del pensamiento racional por sobre el sentir). En su camino por recuperarla (que es un camino de búsqueda de bienestar), accede a la ayahuasca como un camino más entre otros (como vimos en la introducción de este artículo generalmente antes de llegar a probar ayahuasca muchas personas pasan por distintas actividades, incluso luego de las tomas siguen participando de estas otras actividades del "circuito alternativo"). Luego de la experiencia, la persona se transforma ontológicamente ya que lo que está en juego son nociones de "persona" y del ser. Es decir, pasan de la dicotomía cartesiana entre mente-cuerpo para sustituirla por la de sentir. De 
esta forma, es la experiencia del sentir, y la atención a ella, lo que permite el desarrollo del "bienestar". Es decir, se "está bien" cuando aprendemos a educar nuestra atención - con la ayuda de las plantas maestras - hacia el sentir, cuando, según la perspectiva nativa, se logra el equilibrio que rompe la separación entre el cuerpo y el pensamiento, convirtiendo al cuerpo y a la persona misma, como afirma Merleau-Ponty, en un "sujeto corporizado" (MERLEAU-PONTY apud JACKSON, 2010). Cabe destacar que esta transformación no siempre es instantánea. Muchos/as ayahuasqueros/as señalan que se produce después de un tiempo de la toma en dónde pueden reflexionar acerca de lo que se les presentó en sus visiones, e incluso otros señalan que les llevó varias tomas de ayahuasca.

Las ceremonias de ayahuasca suscitan la construcción de un mito que es tanto singular como colectivo. Esta singularidad viene dada por el hecho de que son las propias emociones, sentimientos, miedos, traumas, experiencias de vida personal, las que afloran y resurgen en una toma de ayahuasca (el set). Pero también es colectivo (volvemos a lo que al final de la sección anterior mencionábamos como setting y como el contexto y el entorno moldean las propias experiencias singulares) porque suscita un, como mencionaba el fundador del CCT, mito arquetípico. Y esto viene dado, no sólo por las reflexiones en torno a la práctica que se comparten al finalizar una meditación o una ceremonia, sino también por la propia ayahuasca. Esto sucede en dos sentidos. En primer lugar, porque las visiones suelen contener patrones comunes que exceden la historia de vida singular de cada persona. En este sentido, resultan muy interesantes los aportes del psicólogo Benny Shanon quien destaca que las escenas vistas por los bebedores de ayahuasca durante las ceremonias representan narrativas elaboradas y traen mensajes a los que los sujetos atribuyen importancia. Este autor divide los elementos de contenido más comunes (y repetitivos) en las ceremonias de ayahuasca en cuatro categorías, siguiendo un exhaustivo trabajo cualitativo. De esta forma, señala que el primer dominio estaría caracterizado por elementos de la naturaleza. Aquí destaca la presencia de animales (serpientes, reptiles, aves, jaguares), paisajes naturales y escenas de vegetación y jardines. El segundo dominio estaría integrado por elementos de la cultura (ciudades majestuosas, magnificencias de la realeza, varios productos de creación artística, religioso y mágico. Generalmente, según señala, son elementos que no pertenecen al medio sociocultural del propio bebedor, más bien asociados a civilizaciones antiguas). El tercer dominio estaría integrado por elementos de fantasía (tierras mágicas y encantadas habitadas por todo tipo de criaturas que no son ni humanos ni animales; no son escenas mundanas, sino que están asociadas a la mitología y a los cuentos de hadas y magia). El último dominio estaría integrado por elementos espirituales y 
sobrenaturales: personas y reinos celestiales, seres divinos y semidivinos con significados espirituales y metafísicos (SHANON, 2003).

En segundo lugar, estas visiones y experiencias que la ayahuasca suscita traen consigo un sentimiento de unidad entre quienes se encuentran compartiendo ese espacio. De hecho, muchos afirman que aquel que llora o vomita durante la ceremonia no sólo está limpiando y sanando por sí mismo, sino que también lo está haciendo por todos los que se encuentran allí presentes. Aquí se re-significan cuestiones que de manera preliminar podríamos clasificar como "malestar". Me refiero a los efectos fisiológicos de la bebida como podrían ser el mareo, las náuseas o el vómito. Estas cuestiones son re-significadas y se constituyen en partes fundamentales de la sanación. Así, el cuerpo se "limpia" de todo "malestar" (propio y ajeno) a través del vómito. Esta etapa de "malestar" es acompañada del soplado de tabaco (generalmente soplado por los fundadores del CCT o por ayahuasqueros/as más experimentados/as) y del canto (o ícaros), los cuales ayudan a alivianar estos efectos. Luego de esta etapa de limpieza y liberación, denominada por los/las ayahuasqueros/as como la más turbulenta o como remover en el barro, comienza de a poco a asomarse el "bienestar". Después de estos momentos de "malestar", las personas suelen dormir. Es en el momento en el que despiertan que estas sensaciones de mareo, náuseas, angustia o miedo (ocasionados por las propias visiones) desaparecen.

Volvamos un momento más a la cuestión del mito, ya que estas ideas de "bienestar" y "malestar" serán retomadas en la última sección de este artículo. Este mito no aparece como una "creencia", en su sentido más esencialista, como una adherencia ciega, fija a un conjunto de valores morales. Más bien la "creencia” o la adhesión a este mito explicativo es siempre vista como el acto de creer posible, verosímil o verdadera cierta afirmación sobre un estado de cosas que existe en el mundo externo (SEVERI, 2010). Si, al menos en situaciones rituales, estamos predispuestos a creer en algo no es porque compartimos un mismo patrimonio común, una misma cultura, sino porque gran parte del contenido de la creencia es fruto de una proyección privada del observador que cree (SEVERI, 2010). Además, en muchas ocasiones, los vehículos de la creencia son discursos entretejidos de imágenes que se ofrecen a la interpretación personal guiada por el contexto ritual más que reglas impuestas para obedecer (SEVERI, 2010). Entonces, la creencia debe pensarse como un particular proceso proyectivo que conduce al establecimiento de un tipo peculiarmente complejo de vínculo (SEVERI, 2010). No obstante, muchas de estas proyecciones, como vimos con anterioridad, son moldeadas por el entorno en el cual suceden. 
Si las personas relacionan ayahuasca con sentir y con la idea de búsqueda de espiritualidad y bienestar, es porque primero partieron de una relación, un vínculo que el/la ayahuasquero/a construye con la planta de ayahuasca. Рara pensar que la ayahuasca brinda bienestar emocional y espiritual, primero hay que encontrarse con el brebaje en la situación ritual. Luego, será en comunión y diálogo con los demás participantes que estas ideas de bienestar irán tomando forma. Lo mismo se aplica para la búsqueda de bienestar a partir de las meditaciones chamánicas y del soplado de tabaco.

La "creencia" entonces no es fija y estable, no es una esencia. Para Severi, la creencia aparece como un dubitativo, un "no saber si" más que como un afirmativo "saber que". Es decir, nunca se presenta la certeza de que la planta efectivamente pueda sanar en la ceremonia en la que se participe. De hecho, tampoco existe la certeza de que la planta traiga visiones, ya que se han presentado casos de personas en los que la planta nos les hizo efecto alguno (al menos en la primera toma). La duda siempre está presente, no sólo en los/las neófitos/as (que hasta que toman el brebaje "no saben" muy bien qué se les va a presentar durante la ceremonia) sino también entre los/las ayahuasqueros/as más experimentados/as, quienes afirman que muchas veces con la planta nunca se sabe.

Esto nos lleva a la última cuestión a la que se encuentra asociada esta "estética del bienestar" que es la práctica ritual.

\section{NO IMPORTA SI LO QUE ESTÁ SUCEDIENDO ES BUENO O MALO. BIENESTAR Y MALESTAR EN LA SANACIÓN DEL SENTIR.}

La cosa fue así, yo estaba teniendo una crisis bastante aguda por el consumo de alcohol, algunas drogas también, de marihuana, a veces alternativamente cocaína. Venía de un cáncer importante donde no tenía energía ni para levantarme de la cama. Entonces, las drogas más allá de ser una cosa recreativa, era una cosa intuitiva para poder conectarme, estar en el día. Tenía dolores y además estaba deprimida y estaba muy flaquita, pesaba 52 kilos. Entonces, no sólo era (...). por supuesto era una fuga, una analgesia (...). Yo me sentía mal, estaba muy incómoda con lo que me estaba pasando. Entonces, me pareció que era tiempo de buscar porque sino los excesos iban a llegar pronto. Entonces, empecé a buscar en Internet y extrañamente mi mejor amigo había tomado ayahuasca acá y yo lo había acompañado mientras había hecho la dieta, había estado con él, pero no había registrado. O sea, lo había acompañado a él en toda su preparación, que había sido muy mala, por cierto. Y él no tuvo una experiencia muy amorosa. Entonces había quedado en el olvido. En un momento yo le comenté a él que iba a venir a hacer, que quería (...). Bueno tuve esa experiencia y la experiencia fue única. O sea, la sensación de esa primera vez fue sentir una inmensidad... por un lado, sentirme única y por otro lado, sentirme parte del todo. Fue la primera vez que esa hermandad que yo habia imaginado de la humanidad, existía. Ese lugar de amor, de solidaridad, de confianza, de entrega. Esa idea de una humanidad superior que todo lo comprende y a todo lo ama. Esos dos, esos grandes ítems: comprensión y amor. O sea que con esas dos sensaciones podes transitar todo. No importa si lo que está sucediendo es bueno o malo, lo podes comprender y podes sentir amor que no pasa nada (...). Vivis la felicidad con total consciencia de que también se acaba. Entonces, ni estás apegada a los buenos momentos ni estás 
apegado, aferrado, a los malos momentos. Hay algo que se impregna, la planta se impregna en el alma. Hay una cosa de continuidad y de total certeza en el sentir.

Este es el relato, grabado en una charla en abril del 2016, de una mujer de unos 57 años aproximadamente. Lo escogí porque me parece que resume las cuestiones planteadas con anterioridad: la imbricación entre "singularidad" y "unidad", las reflexiones positivas con respecto a la experiencia del bienestar, pero también menciona muy al pasar la cuestión que quisiera tratar en esta sección: la idea de que en la ceremonia también pueden ocurrir momentos malos pero que contribuyen al bienestar. En una ceremonia de ayahuasca, estos momentos clasificados como malos se traducen en malestares físicos como mareos o vómitos, en visiones que pueden asustar o clasificarse como aterradoras. Muchos/as ayahuasqueros/as me han comentado que en determinados momentos de sus ceremonias han visto figuras como demonios que les han parecido un poco tenebrosas; así como también muchos/as se han visto deambulando por cementerios o incluso han sentido que estaban muriendo; otros/as han comentado revivir experiencias de sus vidas personales que han sido traumáticas para ellos/as. Aún cuando estas cuestiones puedan resultar negativas son re-significadas en términos de "bienestar". En esta re-significación es muy importante la figura de los fundadores del centro en tanto guías durante la ceremonia. Es la presencia de un guía, de la protección del tabaco, de la guía de los cantos, lo que genera la confianza en las personas de que aunque la experiencia aparezca como aterradora todo estará bien porque ello es parte de la sanación. La clave, para muchos/as, es tratar de no racionalizar la experiencia mientras ocurre. Рara ello, recomiendan, por ejemplo, centrarse en la respiración.

En mis experiencias con ayahuasca he visto figuras que me han aparecido tenebrosas, incluso he sentido que una parte de mí moría ese día, pero fue la concentración en mi respiración y un sentimiento de protección inmenso los que me han llevado a entender en esos momentos que "todo iba a pasar" y que "todo iba a estar bien". A su vez, he experimentado sentimientos de amor y de gratitud hacia la planta, las personas que estaban conmigo en ambas tomas y las personas más cercanas a mí vida cotidiana: mi familia, mi pareja, mis amigos, incluso mi director de tesis y algunos de mis profesores. Pero fue recién en abril del 2019 que esta relación entre "malestar" en el "bienestar" se me presentó como evidente y clara. Esto sucedió en mi participación en una ceremonia de rape de tabaco.

Muy resumidamente las ceremonias de rape son parecidas a lo que he descrito en otro artículo (2018) como "meditaciones chamánicas". Las diferencias son sólo dos. En primer lugar, las ceremonias de rape duran alrededor de tres horas, las meditaciones sólo duran una hora. La segunda es que incluye la toma de rape. El rape de tabaco es el mismo mapacho utilizado para 
soplar pero en una especie de polvo. Se toma de la siguiente manera: quienes ofician la ceremonia toman unos instrumentos muy parecidos a las pipas de madera pero más finitas y alargadas. Éstas cuentan con dos orificios en sus extremos. Uno sirve para soplar, en el otro (ubicado en la base de la pipa) se pone un poco de rape de tabaco. Este rape será depositado en cada uno de los orificios nasales de la persona que se encuentra meditando. Una vez que el rape es soplado en ambas fosas nasales es muy común sentir una especie de ardor en toda la zona de la nariz, que los ojos lloran o muchas ganas de estornudar. A continuación compartiré algunos fragmentos registrados en mi diario de campo sobre dicha ceremonia.

Antes de comenzar, la fundadora explicó lo que iba a suceder. Dos hombres (que en las meditaciones suelen ayudar a los fundadores con el soplado de tabaco) fueron los encargados de dar el rape. Uno de ellos comenzó por la persona que estaba sentada a la derecha de los instrumentos y fue siguiendo la filita. Llegó a mí. Tomó tabaco con la pipa y sopló por mi nariz. Inmediatamente comencé a sentir el ardor. Y todavía faltaba la otra fosa nasal. Procedió de la misma manera. Comencé a sentirme terriblemente mareada. Mi cuerpo empezó a sudar, mi temperatura se elevaba. Intento darme aire con las palmas de mis manos. Algo necesitaba salir. Veo que enfrente de mí hay un balde. Me abrazo a él y comienzo a vomitar una y otra vez. Un señor, que suele soplar tabaco en las meditaciones chamánicas, cada tanto pasaba y me acariciaba la espalda. Me soplaba tabaco para aliviar mi sentir. Cuando logro pararme me dirijo hacia el hall. Me siento en un banquito. Y sigo vomitando en mi balde. Mientras tanto, en el banco del costado había otra mujer que también estaba con su balde. La escuché decir Creí que esto iba a ser más fácil. En algún punto, pese a mi malestar, su comentario me resultó gracioso. Pensé que tal vez no tenía que ser tan fácil si es que veníamos a sanar. También pensé que la ayahuasca había sido más buena conmigo, ya que no me había provocado vómitos en ninguna de las dos ocasiones en las que la bebí. En cambio, este rape no me la dejó pasar. Mientras tanto el señor cantó para nosotras, para aliviarnos. También mojó mi cabeza y brazos con agua de florida, todo con el mismo objetivo. Luego, una mujer se me acercó (la que da los talleres de chamanismo andino, otra de las actividades que ofrece el CCT) y me dijo entra asi te pueden cantar y te alivias. No podía mover mi cuerpo de la posición en la que me encontraba. Tampoco quería dejar el balde, que lo sentía como mío y que me daba seguridad. Luego de un rato, pude entrar. Antes me dirigí al baño y me encargue de limpiar el balde. Entré a la habitación donde la ceremonia de rape estaba realizándose. Deje el balde, ahora limpio, donde estaba inicialmente y me acosté. Y dormí. Sentí que el fundador del CCT se me acercó. Apoyó sus manos en mi espalda y en mi cabeza. Me cantó emitiendo unos sonidos indescifrables. Ya todo había pasado. Empecé a sentir mucho frío y mi cuerpo comenzó a temblar. Me levanté, busque una manta (en el hall del centro, sobre una silla hay varias mantas que las personas pueden utilizar durante la meditación o las ceremonias en caso de sentir mucho frío) y volví a acostarme. De ahí en más dormí lo que restó de la meditación. Cuando abrí mis ojos, las luces estaban encendidas. Ya había menos gente, muchos/as ya se habían ido. Mientras doblo la manta que usé para protegerme del frío que mi cuerpo sentía, el hombre que estuvo meditando a mi lado me pregunta ¿Te sentís bien? Le sonrío; Sí, ya me siento mucho mejor. Era muy fuerte ese rape. - Sí, yo también vomité un poquito en un momento. ¿Era la primera vez que tomabas? - No, ya había tomado dos veces antes pero nunca me había pasado así. - ¿Habías tomado ayahuasca? - Sí, dos veces. La primera vez en 2017 y la segunda vez en agosto del 2018. Me saluda porque tiene que irse. Voy a guardar la mantita a su lugar. Busco al señor que estuvo conmigo durante todo el proceso. Siento necesidad de agradecerle por sus cuidados. Lo veo hablando con la chica del chamanismo andino. Me dirijo hacia ellos; Quería agradecerte por todo el cuidado. En serio, muchas gracias. Me sonríe. No, por favor. ¿Te sentís mejor? A todos nos pasó y me dice una frase que en ese momento me gustó mucho lo horrible es también maravilloso... Es como el dolor de muelas, te duele y cuando te 
la sacan ya pasó todo... Voy a saludar a uno de los fundadores. Nos abrazamos. ¿Vos eras la chica que estaba acostada allá?- Sí, era yo -. Ah, te dieron mucho rape. Sos muy sensible como yo. Y tu cuerpo es muy chiquito. Tenes que tomar un poquito nada más. Como yo. Un poquito para alcanzar un estado meditativo y listo. Sino no vas a querer tomar más (...).

Esta experiencia que registré en mi diario de campo me hizo entender la importancia de comprender el sentir (y su "estética del bienestar") como sumamente imbricados a la práctica, al estar ahí meditando (sea justamente en una meditación o en una ceremonia de ayahuasca, la cual es entendida como una meditación plena). El sentir encierra dos aspectos que a simple vista podrían aparecer como contradictorios pero que el trabajo de campo sistemático, mis propias experiencias con las plantas de ayahuasca y de tabaco, y una contextualización de los significados ligados a la práctica y comportamiento ritual, muestran como pertenecientes a cierta "lógica" (más bien estética) particular: el "malestar" (mareos, vómitos, visiones aterradoras, miedos personales...) y el "bienestar" (sentimientos calificados como positivos que llevan a sentir un alivio posterior). Así "bienestar" y "malestar" aparecen como parte de lo mismo, se superponen y se complementan. La búsqueda del alivio, de la sanación y del bienestar viene de atravesar cierto "caos". "Lo malo" y "lo oscuro" son momentos que deben ser atravesados para alcanzar la sanación y el bienestar. Es esto lo que llevó a mi cuidador en esa ceremonia de rape a decirme que lo horrible (el vómito) también puede ser maravilloso (una vez que limpié mi cuerpo a través del vomito todo el malestar desapareció porque aquello que me "hacía sentir mal" debía salir de mi cuerpo). Y es esta vinculación también la que llevó al fundador del CCT a entender las experiencias con plantas maestras en términos de mito del héroe. El camino del héroe no es fácil. Antes de llegar a su meta atraviesa una serie de dificultades (malestares físicos, visiones aterradoras), si logra vencerlas (guiándose con su sentir) alcanzará su meta (recuperará su bienestar).

Explicitemos estas cuestiones. Empecemos con el "bienestar". Éste es entendido como sentirse bien y esto sucede cuando se logra la re-conexión con el sentir, es decir, cuando se comprende al cuerpo como sujeto que siente y piensa y piensa y siente. El tabaco, y sobre todo la ayahuasca, aparecen como las guías que ayudan a las personas a equilibrarse. Este equilibrio se traduce como estar presentes, estar atentos al aquí y ahora lo cual implica educar al cuerpo a concentrarse en lo que está haciendo (por ejemplo, meditar). Por su parte, el "malestar" no sólo se reduce a los efectos fisiológicos de la ayahuasca sino que, y sobre todo, refiere a algo anterior a la toma. Aquello que se entiende bajo esta categoría refiere, por un lado, a cuestiones singulares de cada persona (como pueden ser miedos propios, fobias, la muerte de algún ser querido o cualquier experiencia entendida como traumática, el consumo y abuso de sustancias como "drogas", alcohol o cigarrillos, angustias, etc). Por otro lado, refiere a esos arquetipos que 
J comentaba al inicio de esta sección: una angustia o una crisis generalizada inherente al ser humano como consecuencia de su dualismo entre mente y cuerpo. De esta forma, dentro de la perspectiva nativa, retomar el equilibrio entre ambas dimensiones (que, como vimos, se entienden en términos de unidad) permite alcanzar bienestar que para ellos/as, es tanto emocional como espiritual. Esta espiritualidad refiere a la re-construcción de un vínculo con "lo sagrado" que en este caso particular sería con la propia planta de ayahuasca y con las visiones que ella suscita. Se trata de una espiritualidad que, por un lado, se encuentra anclada en el sentir y que por el otro, busca distanciarse de una religión que ven como dogmática y formalista.

La "espiritualidad" se desprende de las "religiones tradicionales", los dogmatismos, la organización jerárquica de su institución y de las tecnologías del pastorado (FOUCAULT, 2008) para convertirse en una "tecnología del yo". Entendidas estas últimas como "juegos de verdad" específicos, como saberes desarrollados por los seres humanos acerca de sí mismos (FOUCAULT, 2008). Las "tecnologías del yo" permiten a los individuos efectuar, por cuenta propia o con la ayuda de otros, cierto número de operaciones sobre su cuerpo y su alma, pensamientos, conductas o cualquier forma de ser, obteniendo así una transformación de sí mismos con el fin de alcanzar cierto estado de felicidad, pureza, sabiduría o inmortalidad (FOUCAULT, 2008). Entre estas tecnologías "el cuidado de sí", "la preocupación por sí", "el sentirse preocupado, inquieto por sí", "el ocuparse de uno mismo", "el cuídate a ti mismo", "el conócete a ti mismo", figuran entre sus principios más importantes (FOUCAULT, 2008). Se trata, de esta manera, de una "espiritualidad" centrada en el auto-conocimiento. Sin embargo, esto ni significa que se centre y promueva el individualismo, ya que esta noción de "espiritualidad" es construida en y a partir de una relación: la relación que cada persona entabla con la planta de ayahuasca, y el diálogo que surge del compartir las experiencias y reflexionar sobre ellas con los demás. Para construir esta "tecnología del yo" hay que partir del hecho de que estos cuerpos habitan un mundo.

Podemos ver entonces que, como afirma Viotti (2018) para el caso de El Arte de Vivir y su concepción de la energía, existe una complementariedad entre técnicas corporales y técnicas espirituales. De hecho, podríamos afirmar que la técnica corporal terapéutica no puede pensarse por fuera de la espiritualidad. La dimensión terapéutica de la espiritualidad se mantiene dentro de criterios complementarios y dentro de un horizonte en el que las causas del bienestar y el malestar son tanto naturales (vinculadas a fenómenos corporales, físicos y biológicos) como psicosociales (asociadas con estilos de vida y conductas que tienen que ver con el cuidado de uno mismo y su entorno) (VIOTTI, 2018). Tanto el "malestar" como el "bienestar" y la "espiritualidad", en el CCT, se encuentran atravesados por una lectura claramente psicologizada. Como afirma Pablo Semán la noción de psicologización implica por un lado, el uso de un recurso 
psicoterapéutico y el desenvolvimiento de categorías que problematizan y valorizan el procedimiento de una búsqueda interior (SEMÁN, 2000). A diferencia del uso de la función psicoterapéutica, la psicologización implica un discurso cuya significación es localizada: se trata de sujetos que no agotan su experiencia en el "yo" sino que no dejan de pensarse como dependientes de una orden divina en la que viven y postulan como antecedente lógico y cronológico y también experiencial: la construcción de una interioridad se marca en el contexto de un círculo encantado del cual el sujeto sigue constituyendo (SEMÁN, 2000). Es decir, el bienestar espiritual supone una trama más extensa que la del propio cuerpo (VIOTTI, 2018). Una trama que para el CCT incluye sentires interconectados (el del propio cuerpo como el de los otros) y plantas que ayudan a esa interconexión.

Como mencioné al principio de este artículo siguiendo a Birgit Meyer, la "estética" no pude ser considerada como sinónimo de "lo bello", "lo armonioso" del arte. Sino como la relación entre nuestros sentidos con el mundo en el que habitamos. En el caso particular que aquí presenté, la "estética del bienestar" designa la relación del sentir de las personas con el mundo que habitan. Y el sentir, su desarrollo y el re-encuentro de las personas con él, se encuentra sumamente vinculado a las experiencias con ayahuasca y con tabaco, ambas plantas entendidas como medicinales, sanadoras del malestar emocional y espiritual. El sentido de la estética del CCT se encuentra sumamente ligado a sus experiencias con las plantas de ayahuasca y el mapacho. No surge de una representación, no son metáforas, no son ideas que sus mentes han creado aisladamente, son más bien índice de una trama de relaciones entre humanos y nohumanos.

\section{CONCLUSIONES}

Рara comprender qué se entiende por "bienestar", "espiritualidad" y "malestar" en el CCT es necesario remitirse a la categoría del sentir. Como no se trata de una categoría que cuente con una definición formal, para entenderla es necesario remitirse a su relación con las ceremonias de ayahuasca y con las meditaciones de tabaco. A partir de entenderla en acción y como resultado de una relación con las plantas maestras, el sentir va adquiriendo sentidos que los fundadores del centro y las personas que participan de sus actividades le van atribuyendo. Sentidos que deben entenderse desde la experiencia como la relación entre ciertas lecturas (en los fundadores del CCT influenciadas por sus carreras de psicólogos) y la práctica (la situación de encuentro con las plantas maestras en las ceremonias de ayahuasca o de rape).

De esta manera, de acuerdo a lo expresado en este artículo, el sentir aparece como una categoría abarcadora de experiencia corporal que no se puede explicar con palabras y que, 
además, debe guiar la acción de las personas. También vimos que esta categoría no está sola, sino que es entendida a partir de su contra cara, el pensamiento racional. No obstante, esta oposición que a simple vista parecería irreconciliable en realidad constituye más bien una estrategia discursiva que sirve para explicar con palabras una categoría (el sentir) estrechamente vinculada con las emociones y los sentimientos del cuerpo. Para el CCT sentir y pensamiento racional no son contradictorios, sino que deberían complementarse. Ambos, en conjunto, son constitutivos de la persona. Pero para el CCT el problema radica en el hecho de que nuestra cultura sólo puso énfasis en uno de ellos, en el pensamiento racional, y olvidó o dejó de lado el sentir. Esto es lo que para ellos/ellas producen malestar y crisis en las personas. Y es a partir de aquí, de esta crisis y desequilibrio de énfasis, que construyen un mito. Es decir, una narrativa que parte de esa crisis existencial y que lleva a una búsqueda que culmina con el encuentro entre la persona y la planta de tabaco o de ayahuasca (o con ambas). A partir de esa situación de encuentro las personas establecen un tipo particular de vínculo con estas plantas maestras, una relación de bienestar emocional y espiritual (que implica un paso por el malestar) interpretada en clave psicologizada. Como vimos en la última sección de este artículo, los sujetos no agotan su experiencia en el "yo" sino que la construcción de su interioridad se marca en el contexto de un círculo encantado del cual el sujeto sigue constituyendo (SEMÁN, 2000).

Pero estas plantas suelen tener, en el momento de la ingesta, efectos en nuestro cuerpo que podrían ser calificados de manera negativa (como malos o feos). En el caso de la ayahuasca, vimos que en algunas ocasiones y para algunas personas, se pueden experimentar vómitos, descomposturas, mareos. Así como también puede traer visiones que pueden resultar aterradoras. En el caso del rape de tabaco podemos experimentar ardor en la nariz, estornudos, lagrimeado en los ojos, un poco de toz, incluso vómitos y mareos. Sin embargo, estos efectos a primera vista negativos (vomitar por ejemplo nos puede parecer una acción un tanto desagradable y hacerlo en público puede parecernos un poco vergonzoso) son re-significados en términos de bienestar. El malestar y el bienestar no aparecen en una relación de causa y efecto de tipo lineal, sino más bien en una relación de tipo circular: ambos se están imbricando en la experiencia ritual.

En este sentido, el presente artículo pretendió mostrar la importancia de entender las categorías nativas (el sentir) dentro de los contextos en los que son usadas y creadas (en este caso, ritual). Así como también, siguiendo el planteo de Csordas, que estos "modos somáticos de atención" están culturalmente constituidos. Para el caso particular del CCT, el sentir - como categoría nativa que expresa y alcanza el bienestar - es aquello a lo que se puede acceder mediante la ingesta de ayahuasca, el soplado de tabaco o la toma de rape. Y es a partir de esa 
experiencia y de las reflexiones que las personas hacen en torno a esas experiencias que adquiere su sentido. Así la "estética del sentir" incluye en sí misma aspectos que a simple vista nos parecen contradictorios (sentir/pensamiento racional; bienestar/malestar) pero que la experiencia del sujeto corporalizado muestra como complementarios.

\section{AGRADECIMIENTOS}

Quisiera agradecer al Dr. Rolando Silla, mi director de tesis, por su guía durante todos estos años y especialmente por sus comentarios al primer borrador de este artículo.

A Sylvia y Jorge, mis "maestros del sentir". A todos/as los/as ayahuasqueros/as que han compartido sus experiencias conmigo, especialmente a mi cuidador en aquella ceremonia de rape cuya frase constituye el título de este artículo.

Al Dr. Rodrigo Toniol por la invitación a participar de este dossier. A los evaluadores de la Revista Mundaú cuyos comentarios me han sido de gran ayuda, no sólo para presentar una versión más completa de este artículo, sino que también me han brindado nuevas reflexiones y preguntas para el desarrollo de mi investigación.

\section{REFERENCIAS}

GONZÁLEZ, J. C. A. La psicología analítica de Jung y sus aportes a la psicoterapia. Universitas Psychologica, v. 3, n. 1, enero-junio, p. 55-70, 2004.

APUD, Ismael. El indio fantasmal es reclutado en la ciudad. Neochamanismo, sus orígenes y su llegada a Uruguay. Cuadernos de Antropología Social, n. 38, p. 57-83, 2013.

BELL, Catherine M. "'The Chinese believe in spirits': belief and believing in the study of religion". In: FRANKENBERRY, N. Radical Interpretation in Religion. Cambridge: Cambridge University Press. 2004. p. 100-116.

CAROZZI, María Julia. Nueva Era y Terapias Alternativas. Construyendo significados en el discurso y la interacción. Buenos Aires: EDUCA, 2000.

CSORDAS, Thomas. "Modos somáticos de atención”. In: CITRO, Silvia. Cuerpos plurales: antropología de y desde los cuerpos. Buenos Aires: Biblios, 2010. p. 83-104.

DE LA TORRE, Renée; MARTíN, Eloísa. Religious Studies in Latin America. Annual Review of Sociology, v. 42, p. 473-492, 2016.

DEL RIO, Maria Soledad. 'Psicologización de la espiritualidad': cruces entre psicología, vegetalismo amazónico y antropología en un Centro Comunitario y Terapéutico de la Ciudad de Buenos Aires. Ciencias Sociales y Religión/ Ciências Sociais e Religião, Porto Alegre, v. 20, n. 28, p. 158-171, 2018. 
DUARTE, Luiz Fernando Dias. Antropología y psicoanálisis: retos de las ciencias románticas en el siglo XXI. Revista Culturas Psi, v. 1, n. 1, p. 45-63, 2013.

FOUCAULT, Michel. Tecnologías del yo y otros textos afines. $1^{\text {a }}$ ed. Paidós: Buenos Aires, 2008.

GIUMBELLI, Emerson; TONIOL, Rodrigo. "What is spirituality for? New relations between religion, health and public spaces". In: BLANES, Ruy; MAPRIL, Jose; GIUMBELLI, Emerson; WILSON, Erik (orgs). Secularisms in a Postsecular Age? Religiosities and Subjectivities in Comparative Perspective, 2017. p. 147-167.

GIUMBELLI, Emerson; RICKLI, Joao; TONIOL, Rodrigo. Como as coisas importam. Uma abordagem material da religião. Textos de Birgit Meyer. Ed. UFRGS: Porto Alegre, 2018.

INGOLD, Tim. Conociendo desde dentro: reconfigurando las relaciones entre la antropología y la etnografía. Etnografías Contemporáneas, v. 2, n. 2, p. 218-230, 2015.

JACKSON, Michael. "Conocimiento del cuerpo". In: CITRO, Silvia. Cuerpos plurales: antropología de y desde los cuerpos. Buenos Aires: Biblios, 2010. p. 59- 82.

KUYPERS, K. P. C; RIBA, J.; DE LA FUENTE REVENGA, M.; BARKER, S.; THEUNISSEN, E. L.; RAMAEKERS, J. G. Ayahuasca enhances creative divergent thinking while decreasing conventional convergent thinking. Psychopharmacology, v. 233, n. 18, p. 3395-3403, 2016.

LUHRMANN, Tanya et al. Differences in voice-hearing experiences of people with psychosis in the USA, India and Ghana: interview-based study. The British Journal of Psychiatry, v. 206, n. 1, p. 41-44, 2015.

OLMOS ÁLVAREZ, Ana L. Entre médicos y sanadores: gestionando sentidos y prácticas sobre el proceso de salud-enfermedad-atención en un movimiento carismático católico argentino. Salud Colectiva, v. 4, n. 2, p. 225-240, 2018.

PITT-RIVERS, J. El análisis del contexto y el 'locus' del modelo. Tres ensayos de antropología estructural. Cuadernos Anagrama: Barcelona, 1973.

SCHULTES, Richard Evans; HOFMANN, Albert; RALSCH, Christian. Plantas de los Dioses. Las fuerzas mágicas de las plantas alucinógenas. Fondo de Cultura Económica: México, 2000.

SEMÁN, Pablo. A "Fragmentação do cosmos": um estudo sobre as sensibilidades de fiéis pentecostais e católicos de um bairro de Grande Buenos Aires. Tesis de doctorado. Universidade Federal do Rio Grande do Sul, Porto Alegre. 2000.

SEMÁN, Pablo; VIOTTI, Nicolás. New Age Spirituality in Argentina: Cultural Change and Epistemological Challenge. International Journal of Latin American Religions, v. 3, n. 1, p. 193-211, 2018.

SEVERI, Carlo. "Memoria, proyección, creencia o la metamorfosis del enunciador". In: SEVERI, Carlo. El sendero y la voz. Una antropología de la memoria. Grupo Ed. Sb. $1^{\text {a }}$ Ed. Buenos Aires, 2010. p. 219-172.

SHANON, Benny. Os conteúdos das visões da ayahuasca. Mana, v. 9, n. 2, p. 109-152, 2003. 
STEIL, Carlos Alberto; TONIOL, Rodrigo. "A crise do conceito de religião e sua incidência sobre a antropologia". In: GIUMBELLI, Emerson; BÉLIVEAU, Verónica Gimenéz (Org.). Religión, cultura y política en las sociedades del siglo XXI. 1ed. Buenos Aires: Biblos, 2013. p. 137-158.

STRATHERN, Marilyn. The Gender of the gift: problems with women and problems with society in Melanesia. Berkeley: California University Press, 1988.

TONIOL, Rodrigo. Alternative Therapies. Encyclopedia of Latin American Religions, p. 01-05, 2015. DOI 10.1007/978-3-319-08956-0_3-1

VIOTTI, Nicolás. Más allá de la terapia y la religión: una aproximación relacional a la construcción espiritual del bienestar. Salud Colectiva, v. 14, n. 2, p. 241-256, 2018.

Recebido em 15 de maio de 2019. Aprovado em 07 de outubro de 2019. 